\title{
Random Weighting Estimation for Fusion of Multi-Dimensional Position Data
}

\author{
Shesheng Gao ${ }^{\mathrm{a}}$, Yongmin Zhong ${ }^{\mathrm{b} *}$, Bijan Shirinzadeh ${ }^{\mathrm{c}}$ \\ ${ }^{a}$ School of Automation, Northwestern Polytechnical University, Xi'an, China \\ ${ }^{\mathrm{b}}$ Department of Mechanical Engineering, Curtin University of Technology, Australia \\ ${ }^{\mathrm{c}}$ Department of Mechanical and Aerospace Engineering, Monash University, Australia
}

\begin{abstract}
This paper adopts the concept of random weighting estimation to multi-sensor data fusion. It presents a new random weighting estimation methodology for optimal fusion of multi-dimensional position data. A multi-sensor observation model is constructed for multi-dimensional position. Based on this observation model, a random weighting estimation algorithm is developed to estimate position data from a single sensor. Using the random weighting estimations from each single sensor, an optimization theory is established for optimal fusion of multi-sensor position data. Experimental results demonstrate that the proposed methodology can effectively fuse multi-sensor dimensional position data, and the fusion accuracy is much higher than that of the Kalman fusion method.
\end{abstract}

Keywords: random weighting estimation, multi-sensor system, data fusion, and multi-dimensional position data.

\section{Introduction}

Multi-sensor data fusion is a technique, which aims to combine data from multiple sensors to achieve improved accuracies and more specific inferences than could be achieved by using a

*Corresponding author. Postal address: Department of Mechanical Engineering, Curtin University of Technology, GPO Box U1987, Perth, WA 6845, Australia. Tel: +61 392667657 Fax: +61 392662681 Email: Y.Zhong@curtin.edu.au 
single sensor alone. Although there has been a significant amount of research reported in this area during the past twenty years, fusion of multi-sensor data is still a challenging problem [8, 9, 17]. Currently, the commonly used methods such as Kalman filter [1, 2, 15, 16], Bayesian reasoning $[3,12,19]$ and fuzzy logic theory $[13,14,18]$ suffer from their own limitations in achieving optimal fusion. Such limitations include the dependence on a conditional probability distribution or fuzzy membership function, the unacceptable fusion results when observational evidences highly conflict with each other, the low real-time performance due to the use of too many state variables, and the low efficiency for fusion of multi-sensor information $[8,10,11]$. Random weighting is an emerging computational method in statistics, and has been used to solve different problems [4-7]. In comparison with the existing data fusion methods, such as the commonly used Kalman filter, this method has many advantages [4, 7, 20]. The random weighting method is simple in computation and suitable for large samples. It does not rely on the knowledge of the distribution of position parameters, and the estimation results are unbiased. It can also be used to calculate a statistical probability density function, since the resultant statistical distribution actually provides for a probability density function. Nevertheless, there has been little research to use random weighting estimation for multi-sensor data fusion. To the best of our knowledge, this is the first study focusing on multi-sensor data fusion by using random weighting estimation.

By adopting the concept of random weighting estimation to multi-sensor data fusion, this paper presents a new random weighting estimation methodology for fusion of multi-dimensional position data. This methodology achieves the optimal fusion of multi-dimensional position data based on the random weighting estimations of observations from single sensors. It features a 
multi-sensor observation model for multi-dimensional position, a random weighting estimation algorithm for estimation of single-sensor position data, and a random weighting estimation theory for optimal fusion of the data estimated from each single sensor. Experiments and comparison analysis have been conducted to comprehensively evaluate the performance of the proposed methodology for fusion of multi-dimensional position data.

\section{Random Weighting Estimation for Fusion of Multi-dimensional Position Data}

Assume that $X_{1}, X_{2}, \cdots, X_{n}$ are the random variables of independent and identical distribution with common distribution function $F(x)$, and the corresponding empirical distribution function is defined as $F_{n}(x)=\frac{1}{n} \sum_{k=1}^{n} I_{\left(X_{k} \leq x\right)}$. Let $x_{1}, x_{2}, \cdots x_{n}$ be the corresponding observed realizations. Further, we shall denote $\tilde{X}=\left(X_{1}, X_{2}, \cdots X_{n}\right)$ and $\tilde{x}=\left(x_{1}, x_{2}, \cdots x_{n}\right)$. Then, the random weighting estimation of $F_{n}$ can be defined as

$$
H_{n}(x)=\sum_{k=1}^{n} v_{k} I_{\left(X_{K} \leq x\right)}
$$

where $I_{\left(X_{i} \leq x\right)}$ is the characteristic function, i.e. $I_{\left(X_{k} \leq x\right)}=\left\{\begin{array}{cc}1 & X_{k} \leq x \\ 0 & X_{k}>x\end{array}\right.$. Random vector $\left(v_{1}, v_{2} \cdots, v_{n}\right)$ obeys Dirichlet distribution $D(1, \cdots, 1)$, that is, $\sum_{k=1}^{n} v_{k}=1$ and the joint density function of $\left(v_{1}, v_{2} \cdots, v_{n}\right) \quad$ is $f\left(v_{1}, v_{2} \cdots, v_{n}\right)=\Gamma(n)$, where $\quad\left(v_{1}, v_{2} \cdots, v_{n}\right) \in D_{n}$ and $D_{n-1}=\left\{\left(v_{1}, v_{2}, \cdots, v_{n-1}\right): v_{k} \geq 0, k=1, \cdots, n-1, \sum_{k=1}^{n-1} v_{k} \leq 1\right\}$. 


\subsection{Multi-sensor observation model for multi-dimensional position}

Suppose that a multi-sensor system consists of $N$ sensors $\left\{S_{i}, 1 \leq i \leq N\right\}$. The sampling result may be written as

$$
Z_{i k}=\omega_{i}+\Delta \theta_{i}+\bar{\theta}+e_{i k}
$$

where $Z_{i k}$ represents the $k$ th $\left(1 \leq k \leq n_{i}\right)$ observation value obtained from the $i$ th sensor $S_{i}$, $\bar{\theta}$ represents the true position parameter, and $\bar{\theta} \in \Theta\left(\Theta \subset R^{P}, P \geq 2\right)$. $\omega_{i}$ represents the deviation of sensor $S_{i}$ with respect to a common origin. In most cases, the deviation of a sensor is a constant. $e_{i k}$ is the noise for the $k$ th observation value from sensor $S_{i}$. It is assumed that random vector $\left\{e_{i k}, 1 \leq k \leq n_{i}\right\}$ obeys the independent and identical distribution, and observation noises of different sensors are independent of each other. $\theta_{i}$ represents the position parameter of sensor $S_{i}$, and $\Delta \theta_{i}$ represents the uncertainty for the position of sensor $S_{i}$. The position uncertainty of a sensor is independent of the observation noise of this sensor. The position uncertainties and observation noises of different sensors are also independent of each other, respectively.

Since a non-zero deviation vector can be deducted from observation vector $\left\{Z_{i k}, 1 \leq k \leq n_{i}, 1 \leq i \leq N\right\}$, it can assumed that the given deviations $\left\{\omega_{i}, 1 \leq i \leq N\right\}$ are a zero vector without the loss of generality. Accordingly, (2) may be written as

$$
Z_{i k}=\Delta \theta_{i}+\bar{\theta}+e_{i k}
$$


where each element of $\Delta \theta_{i}$ is bounded, and $1 \leq i \leq N$.

Position uncertainty $\Delta \theta$ is generated due to the disturbance to true position parameter $\bar{\theta}$ in the environment. The disturbance, which is random, is contained in each sensor. Consequently, the value of $\Delta \theta$ for a sensor is either positive or negative. Without the loss of generality, we can assume the mean of $\Delta \theta$ is zero. Since position uncertainties of each sensor are independent of each other, variance matrix $\operatorname{Var}(\Delta \theta)$ is a diagonal matrix whose diagonal elements are limited positive values. Further, since the disturbance in the environment is random, $\Delta \theta$ is related to the uncertainty of the environment. Obviously, $\theta_{i}=\bar{\theta}+\Delta \theta_{i}$ is also a random variable, and $E\left(\theta_{i}\right)=\bar{\theta}$ and $\operatorname{Var}\left(\theta_{i}\right)=\operatorname{Var}\left(\Delta \theta_{i}\right)$. Therefore, the observation model, i.e. (3), can be simplified as

$$
Z_{i k}=\theta_{i}+e_{i k} \quad\left(k=1,2, \cdots, n_{i}, \quad i=1,2, \cdots, N\right)
$$

where $e_{i k}$ is a noise vector with zero mean, and each element of variance matrix $\operatorname{Var}(\Delta \theta)$ $\operatorname{Var}\left(\Delta \theta_{i}\right)$ is an observation noise whose value is bounded positive.

\subsection{Random weighting estimation of multi-dimensional position data}

Let $Z_{i}=\left[Z_{i 1}, Z_{i 2}, \cdots, Z_{i n_{i}}\right]$, where $Z_{i}$ is a $p \times n_{i}$ matrix, and $n_{i} \geq 2$ and $p \geq 2$. Denote $\hat{\theta}_{i}$ as the random weighting estimation for the mean of position parameter $\theta_{i}$, and $\hat{s}_{i i}$ as the random weighting estimation for the variance of $\theta_{i}$. Thus, $\hat{\theta}_{i}$ and $\hat{s}_{i i}$ can be obtained by estimation of each sensor's data $Z_{i}$ by the following algorithm: 
(i) Set the tolerance $\sigma$.

(ii) Calculate the mean of $Z_{i k}$

$$
\bar{Z}_{i k}=\frac{1}{n_{i}} \sum_{k=1}^{n_{i}} Z_{i k}
$$

Calculate the variance of $Z_{i k}$

$$
\operatorname{Var}\left(Z_{i k}\right)=\frac{1}{n_{i}} \sum_{k=1}^{n_{i}}\left[Z_{i k}-\bar{Z}_{i k}\right]\left[Z_{i k}-\bar{Z}_{i k}\right]^{T}
$$

(iii) Calculate the random weighting estimation of $\bar{Z}_{i k}$

$$
\hat{Z}_{i k}=\sum_{k=1}^{n_{i}} v_{k} Z_{i k}
$$

Calculate the random weighting estimation of $\operatorname{Var}\left(Z_{i k}\right)$

$$
\hat{\operatorname{Var}}\left(Z_{i k}\right)=\sum_{k=1}^{n_{i}}\left[v_{k} Z_{i k}-\bar{Z}_{i k}\right]\left[v_{k} Z_{i k}-\bar{Z}_{i k}\right]^{T}
$$

(iv) Calculate the estimation accuracy

$$
D_{i}=\sqrt{\left[Z_{i k}-\hat{Z}_{i k}\right]\left[\hat{\operatorname{Var}}\left(Z_{i k}\right)\right]^{-1}\left[Z_{i k}-\hat{Z}_{i k}\right]}
$$


Then, sort $\left\{Z_{i k}, k=1,2, \cdots, n_{i}\right\}$ in ascending order by the estimation accuracy $D_{i}$, yielding the order statistic $\left\{Z_{i(1)}, Z_{i(2)}, \cdots, Z_{i\left(n_{i}\right)}\right\}$.

(v) Calculate random weighting estimation for the mean of order statistic $Z_{i(k)}$

$$
\hat{Z}_{i(k)}^{*}=\sum_{k}^{n_{i}} v_{k} Z_{i(k)}
$$

Calculate random weighting estimation for the variance of order statistic $Z_{i(k)}$

$$
\hat{\operatorname{Var}}\left(Z_{i(k)}^{*}\right)=\sum_{k=1}^{n_{i}}\left[v_{k} Z_{i(k)}-\bar{Z}_{i k}\right]\left[v_{k} Z_{i(k)}-\bar{Z}_{i k}\right]^{T}
$$

(vi) If $\left|\hat{Z}_{i(k)}^{*}-Z_{i(k)}\right|<\sigma \quad\left(k=1,2, \cdots, n_{i}\right)$, the estimation process is terminated. Otherwise, adjust $v_{k}$ and go to (iii).

Finally, we have $\hat{\theta}_{i}=\hat{Z}_{i(k)}^{*}$ and $\hat{s}_{i i}=\hat{\operatorname{Var}}\left(Z_{i(k)}^{*}\right)$. Further, it can be easily obtained that $E\left(\hat{\theta}_{i}\right)=\theta_{i}$, which means $\hat{\theta}_{i}$ is the unbiased estimation of $\theta_{i}$. It should be noted that in the case that the random weighting estimations $\hat{\theta}_{i}$ and $\hat{s}_{i i}$ cannot be achieved within the given tolerance $\sigma$, the tolerance $\sigma$ is enlarged to re-calculate the random weighting estimations.

\subsection{Optimal fusion estimation}

Section 2.2 provided the computational process for obtaining $N$ independent unbiased estimates $\hat{\theta}_{i}$ and variance estimates $\hat{s}_{i i}(i=1,2, \cdots, N)$ from $N$ sensors. In this section, a 
method for optimal fusion of the $N$ estimates will be discussed.

Let

$$
\begin{gathered}
\hat{\theta}^{*}=w_{1} \hat{\theta}_{1}+w_{2} \hat{\theta}_{2}+\cdots+w_{N} \hat{\theta}_{N} \\
w_{1}+w_{2}+\cdots+w_{N}=I
\end{gathered}
$$

where $w_{i}(i=1,2, \cdots, N)$ is a weighting matrix, and $I$ is a unit matrix.

The optimal fusion estimation is achieved in the sense that $\hat{\theta}^{*}$ satisfies the following conditions:

(a) $\hat{\theta}^{*}$ is the unbiased estimation of $\bar{\theta}$, i.e. $E\left(\hat{\theta}^{*}\right)=\bar{\theta}$;

(b) $\hat{\theta}^{*}$ makes $\operatorname{tr}(s)$ reach its minimum, where $s$ is the variance matrix of estimation error, i.e. $s=E\left[\left(\bar{\theta}-\hat{\theta}^{*}\right)\left(\bar{\theta}-\hat{\theta}^{*}\right)^{T}\right]$, and $\operatorname{tr}(s)$ is the trace of $s$.

It can be easily seen that $\hat{\theta}^{*}$ satisfies condition (a). In the following, we discuss how to satisfy condition (b).

When $N=2$, the following equation can be obtained from (12) and (13)

$$
\hat{\theta}^{*}=w_{1} \hat{\theta}_{1}+w_{2} \hat{\theta}_{2}=\hat{\theta}_{1}+w_{2}\left(\hat{\theta}_{2}-\hat{\theta}_{1}\right)
$$

Therefore,

$$
\bar{\theta}-\hat{\theta}^{*}=\left(I-w_{2}\right)\left(\bar{\theta}-\hat{\theta}_{1}\right)+w_{2}\left(\bar{\theta}-\hat{\theta}_{2}\right)
$$


Let

$$
s=E\left[\left(\bar{\theta}-\hat{\theta}^{*}\right)\left(\bar{\theta}-\hat{\theta}^{*}\right)^{T}\right]=\left(I-w_{2}\right) s_{11}\left(I-w_{2}\right)^{T}+2\left(I-w_{2}\right) s_{12} w_{2}^{T}+w_{2} s_{22} w_{2}^{T}
$$

where $s_{i j}=E\left[\left(\bar{\theta}-\hat{\theta}_{i}\right)\left(\bar{\theta}-\hat{\theta}_{j}\right)\right](i, j=1,2)$. If $i=j, \quad s_{i j} \quad$ is a self-covariance matrix. Otherwise, $s_{i j}$ is a cross-covariance matrix.

Choose $w_{2}$ appropriately such that $\operatorname{tr}(s)$ reaches its minimum. Thus, $\frac{\partial s}{\partial w_{2}}=0$, i.e.

$$
\operatorname{tr}\left(-2 s_{11}\left(I-w_{2}^{T}\right)+2 s_{12}-4 s_{12} w_{2}^{T}+2 s_{22} w_{2}^{T}\right)=0
$$

Further, we have

$$
\begin{gathered}
\left(s_{11}-2 s_{12}+s_{22}\right) w_{2}^{T}=s_{11}-s_{12} \\
w_{2}=\left(s_{11}-s_{12}\right)\left(s_{11}+s_{22}-s_{12}-s_{21}\right)^{-1}
\end{gathered}
$$

Substituting (18) into (14) and (16), the following may be obtained

$$
\hat{\theta}^{*}=\hat{\theta}_{1}+\left(s_{11}-s_{12}\right)\left(s_{11}+s_{22}-s_{12}-s_{21}\right)^{-1}\left(\hat{\theta}_{2}-\hat{\theta}_{1}\right)
$$

and 


$$
s=s_{11}-\left(s_{11}-s_{12}\right)\left(s_{11}+s_{22}-s_{12}-s_{21}\right)^{-1}
$$

Since $\left(s_{11}+s_{22}-s_{12}-s_{21}\right)^{-1}$ is a non-negative symmetric matrix,

$$
\left(s_{11}-s_{12}\right)\left(s_{11}+s_{22}-s_{12}-s_{21}\right)^{-1} \geq 0
$$

By (20) and (21), $s \leq s_{11}$. Similarly we can also obtain $s \leq s_{22}$. These demonstrate that the fusion estimation has higher accuracy than the single sensor estimation.

If $\hat{\theta}_{1}$ and $\hat{\theta}_{2}$ are independent of each other, i.e. $s_{12}=s_{21}$, (19) and (20) may be rewritten as

$$
\hat{\theta}^{*}=\left(s_{11}^{-1}+s_{22}^{-1}\right)^{-1}\left(s_{11}^{-1} \hat{\theta}_{1}+s_{22}^{-1} \hat{\theta}_{2}\right)
$$

and

$$
S=\left(s_{11}^{-1}+s_{22}^{-1}\right)^{-1}
$$

Finally, we have the following theorem for optimal fusion of multi-dimensional position data by random weighting estimation.

Theorem For a multi-sensor system consisting of $N$ sensors, assume the random weighting estimations for each sensor's position parameter are $\hat{\theta}_{i}\left(\hat{\theta}_{i}=\hat{Z}_{i}, i=1,2, \cdots N\right)$. Accordingly, the random weighting estimation for the variance of estimation error is $\hat{s}_{i i}=\hat{\operatorname{Var}}\left(Z_{i}\right)$, 
$\hat{s}_{i i}=\hat{\operatorname{Var}}\left(\hat{Z}_{i}\right)$ and $\hat{s}_{i j}=0$ when $i \neq j \quad(i, j=1,2, \cdots, N)$. Then, the optimal estimation for position parameters of the multi-sensor system is

$$
\hat{\theta}^{*}=s \sum_{i=1}^{N} s_{i i}^{-1} \hat{\theta}_{i}
$$

where $s=\left(\sum_{i=1}^{N} s_{i i}^{-1}\right)^{-1}$.

In the following, we will prove the theorem by using the method of mathematical induction.

Proof When $n=2$, it is known based on (22) that the theorem holds.

Assuming that when $n=N-1$, the theorem holds. Therefore,

$$
\begin{aligned}
\hat{\theta}^{*(N-1)} & =s^{(N-1)} \sum_{i=1}^{N-1} s_{i i}^{-1} \hat{\theta}_{i} \\
s^{(N-1)} & =\left(\sum_{i=1}^{N-1} s_{i i}^{-1}\right)^{-1}
\end{aligned}
$$

When $n=N$, it is known that $\hat{\theta}^{*}=w_{1} \hat{\theta}^{*(N-1)}+w_{2} \hat{\theta}_{N}$. Thus, similar to the case when $n=2$, we can obtain

$$
\begin{aligned}
& \hat{\theta}^{*}=s\left[\left(s^{(N-1)}\right)^{-1} \hat{\theta}^{*(N-1)}+s_{N N}^{-1} \hat{\theta}_{N}\right]^{-1} \\
& s=\left[\left(s^{(N-1)}\right)^{-1}+s_{N N}^{-1}\right]^{-1}
\end{aligned}
$$


Then, by (25) and (26), we have

$$
\begin{gathered}
\hat{\theta}^{*}=s\left[\sum_{i=1}^{N-1} s_{i i}^{-1} \hat{\theta}_{i}+s_{N N}^{-1} \hat{\theta}_{N}\right]^{-1}=s \sum_{i=1}^{N} s_{i i}^{-1} \hat{\theta}_{i} \\
s=\left[\left(\sum_{i=1}^{N-1} s_{i i}^{-1}\right)^{-1}+s_{N N}^{-1}\right]^{-1}=\left[\sum_{i=1}^{N} s_{i i}^{-1}\right]^{-1}
\end{gathered}
$$

The proof of the theorem is completed.

\section{Experimental Results and Analysis}

A prototype system has been implemented by using the proposed methodology for fusion of multi-dimensional position data. Experiments have been conducted to comprehensively evaluate and analyze the performance of the proposed methodology. The comparison with the existing multi-sensor data fusion methods is also discussed in this section.

Trials were carried out to evaluate the capability of the proposed method for estimation of the Earth rotational angular velocity. Two laser gyroscopes (Gyro 1 and Gyro 2) are used to measure the Earth rotational angular velocity in the city of Xianyang (Shaanxi Province, China). The latitude of the city is $30.1527^{\circ}$. The sampling cycles of Gyro 1 and Gyro 2 are $60 s$ and $100 s$, respectively. The sampling time for each gyro is $2400 s$. There are 40 data sets sampled from Gyro 1 and 24 from Gyro 2. The estimation of single sensor data (data from Gyro 1) by the proposed methodology is shown in Fig. 1, in which the estimated angular velocity error is within $\left(-0.7 \times 10^{-6}, 0.7 \times 10^{-6} \mathrm{rad} / \mathrm{s}\right)$. Fig. 2 shows the results for fusion of the estimated gyros' data by the proposed methodology. It can be seen that the angular velocity 
error obtained by fusion estimation is within $\left(-0.2 \times 10^{-6}, 0.2 \times 10^{-6} \mathrm{rad} / \mathrm{s}\right)$, which is much smaller than that by the single sensor estimation. This demonstrates that the proposed methodology can effectively fuse multi-sensor data, and the accuracy is much higher than that of the single sensor estimation.

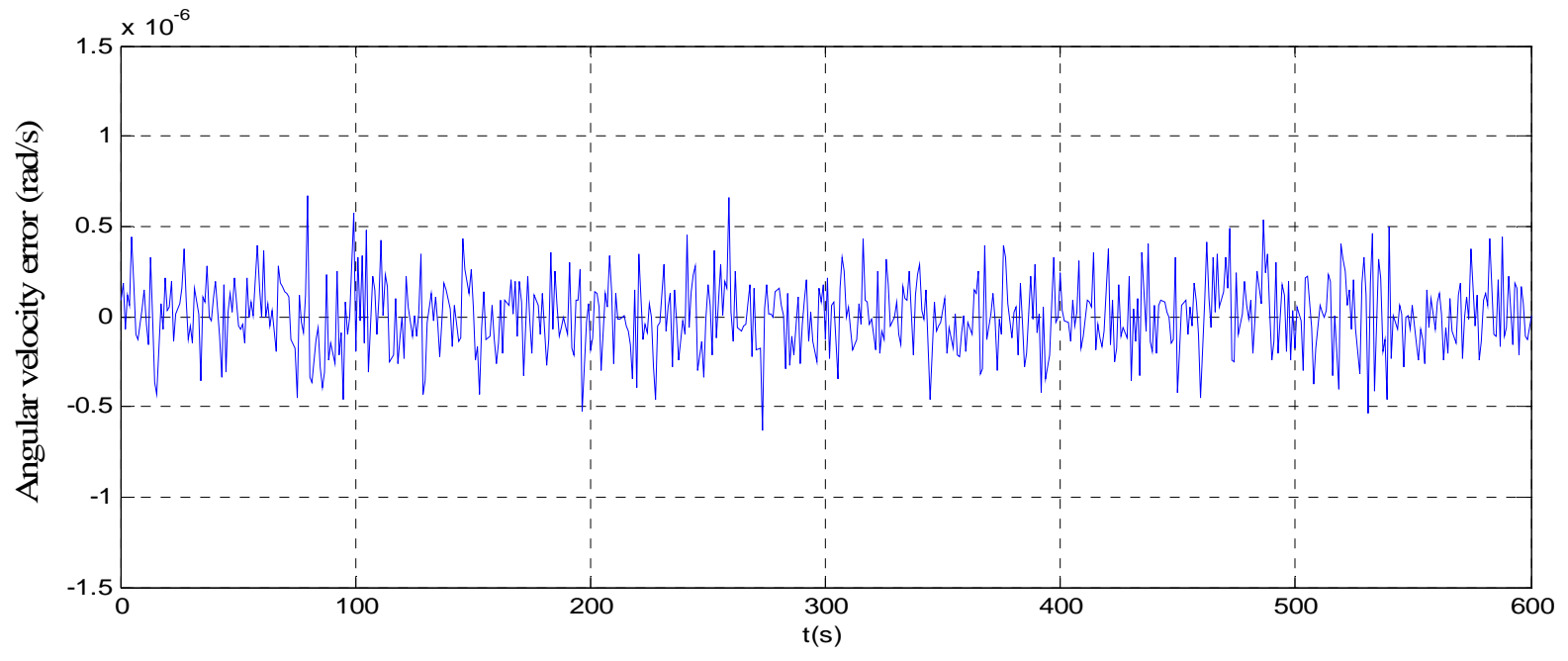

Figure 1. Estimation of single gyro data using the proposed methodology

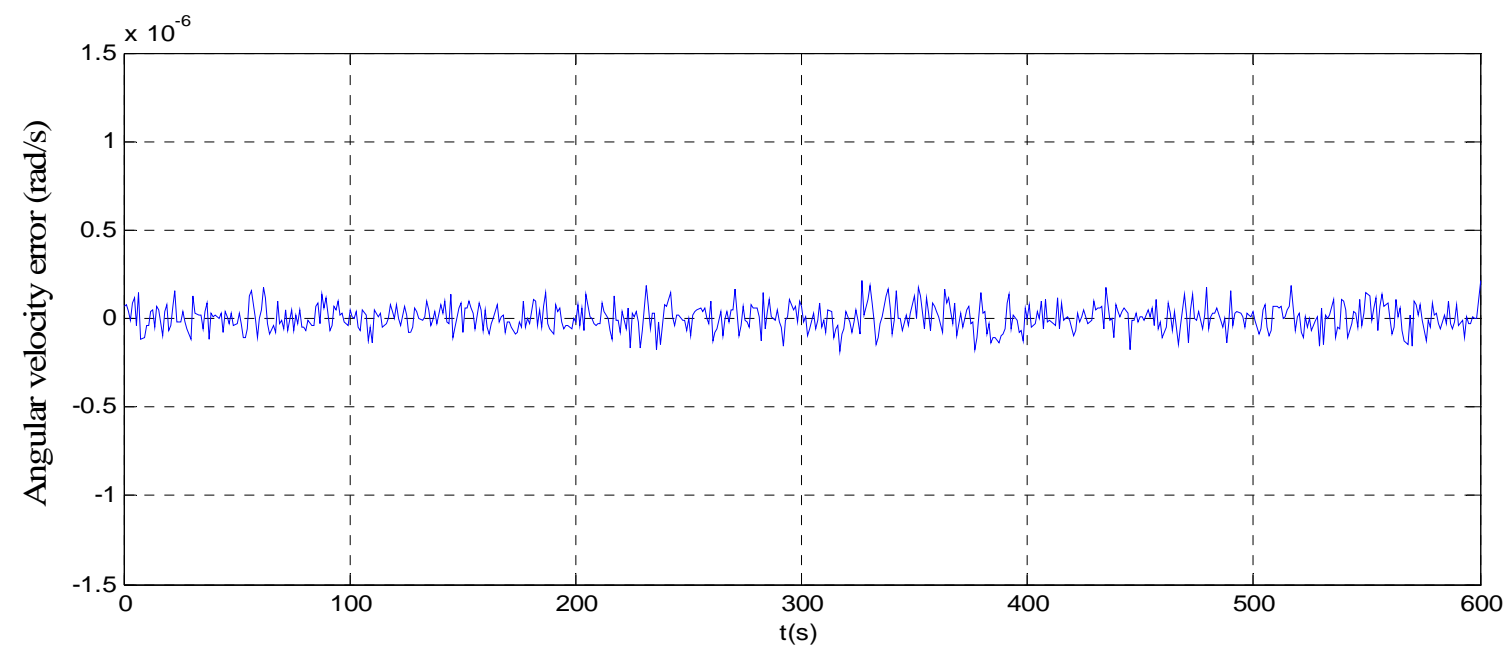

Figure 2. Fusion of the estimated gyros' data using the proposed methodology 


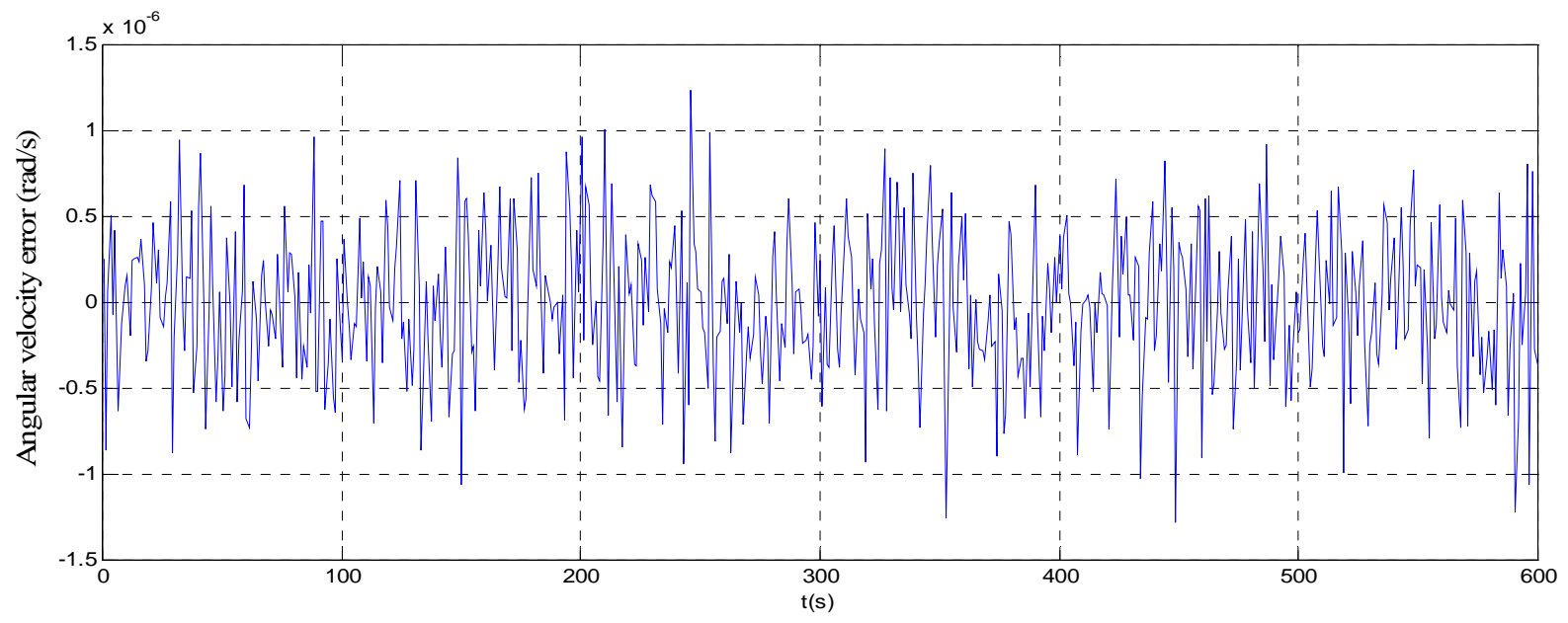

Figure 3. Kalman estimation of single gyro data

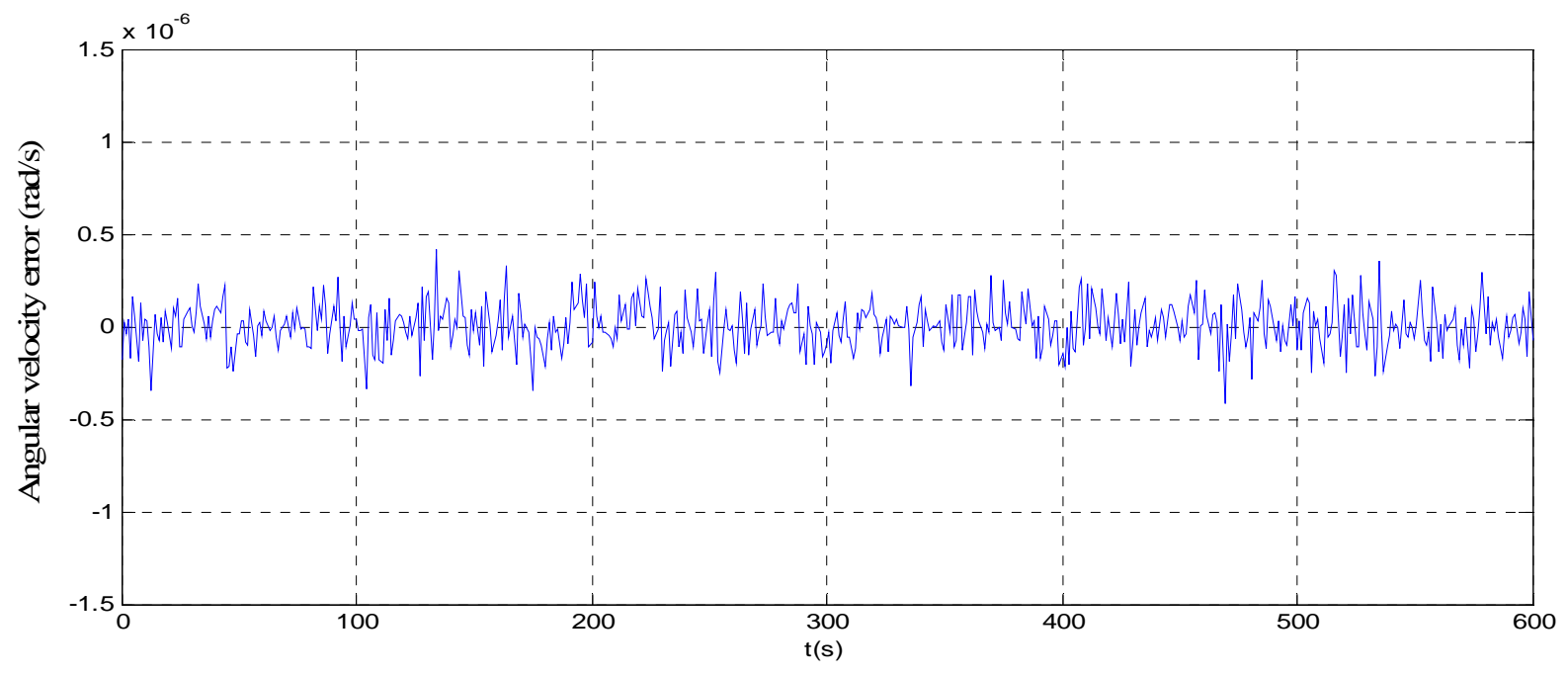

Figure 4. Kalman fusion of the estimated gyros' data

The performance of the proposed fusion method has been evaluated in comparison with the Kalman fusion method. Experiments have been conducted based on the same conditions as Fig. 1 and Fig. 2 by using the Kalman fusion method, and the results are shown in Fig.3 and Fig. 4, respectively. It can be seen from Fig. 3 that the angular velocity error estimated from a single sensor (Gyro 1) by the Kalman method is within $\left(-1.3 \times 10^{-6}, 1.3 \times 10^{-6} \mathrm{rad} / \mathrm{s}\right)$, which is much higher than that obtained by the proposed methodology. As shown in Fig. 4, the angular velocity error obtained by the Kalman fusion of the data estimated from each sensor is within 
$\left(-0.4 \times 10^{-6}, 0.4 \times 10^{-6} \mathrm{rad} / \mathrm{s}\right)$, which is twice higher than that obtained by the proposed methodology. Comparing Fig. 1 with Fig. 3 and Fig. 2 with Fig. 4, it is evident that the proposed method has much higher accuracy than the Kalman method for fusion of multi-sensor data.

Experiments have also been conducted to evaluate the performance of the proposed methodology for fusion of heterogeneous sensor data. A GPS (Global Positioning System) and an accelerometer are used to measure the acceleration of an object moving at a speed greater than $55 \mathrm{~km} / \mathrm{h}$ on a road with a low adhesive coefficient. The moving object has the constant acceleration of $5 \mathrm{~m} / \mathrm{s}^{2}$. For the accelerometer, the zero bias is $10^{-4} \mathrm{~g}$ and the random white noise is $50 \times 10^{-3} \mathrm{~g}$. The sampling time periods for the GPS and accelerometer are $1 s$ and $2 s$, respectively. The dynamic simulation time is 1000s. There are 1000 and 500 data sets sampled from the GPS and accelerometer, respectively. Fig. 5 shows the estimation of the data obtained from a single sensor (the GPS) by the proposed methodology. The maximum error within the time period from $200 s$ to $600 s$ is around $0.1 \mathrm{~m} / \mathrm{s}^{2}$, and the maximum error after $600 \mathrm{~s}$ is around $0.05 \mathrm{~m} / \mathrm{s}^{2}$. Fig. 6 illustrates the results for fusion of the data estimated from both the GPS and accelerometer by the proposed methodology. The maximum error within the time period from 200s to $600 s$ was found to be $0.04 \mathrm{~m} / \mathrm{s}^{2}$, and the maximum error after $600 \mathrm{~s}$ was found to be $0.007 \mathrm{~m} / \mathrm{s}^{2}$. By comparing Fig. 5 with Fig. 6, it can be seen that the accuracy of the fusion estimation is much higher than that of the single sensor estimation. This also indicates that the proposed methodology can effectively fuse heterogeneous sensor data. 


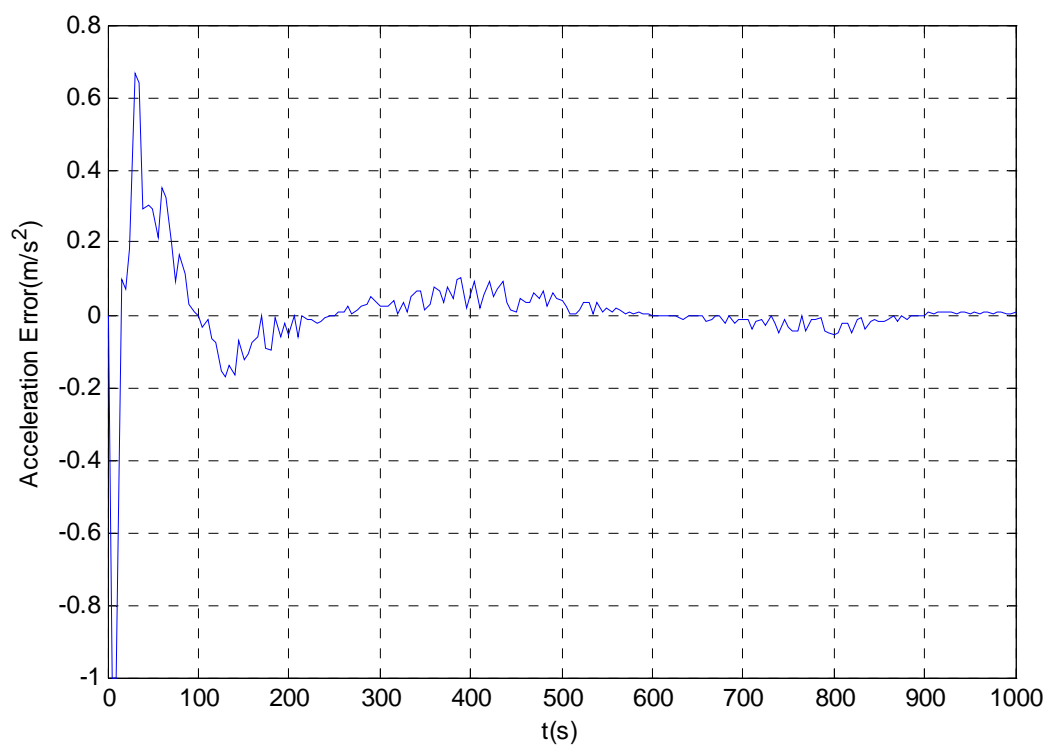

Figure 5. Estimation of the GPS data using the proposed methodology

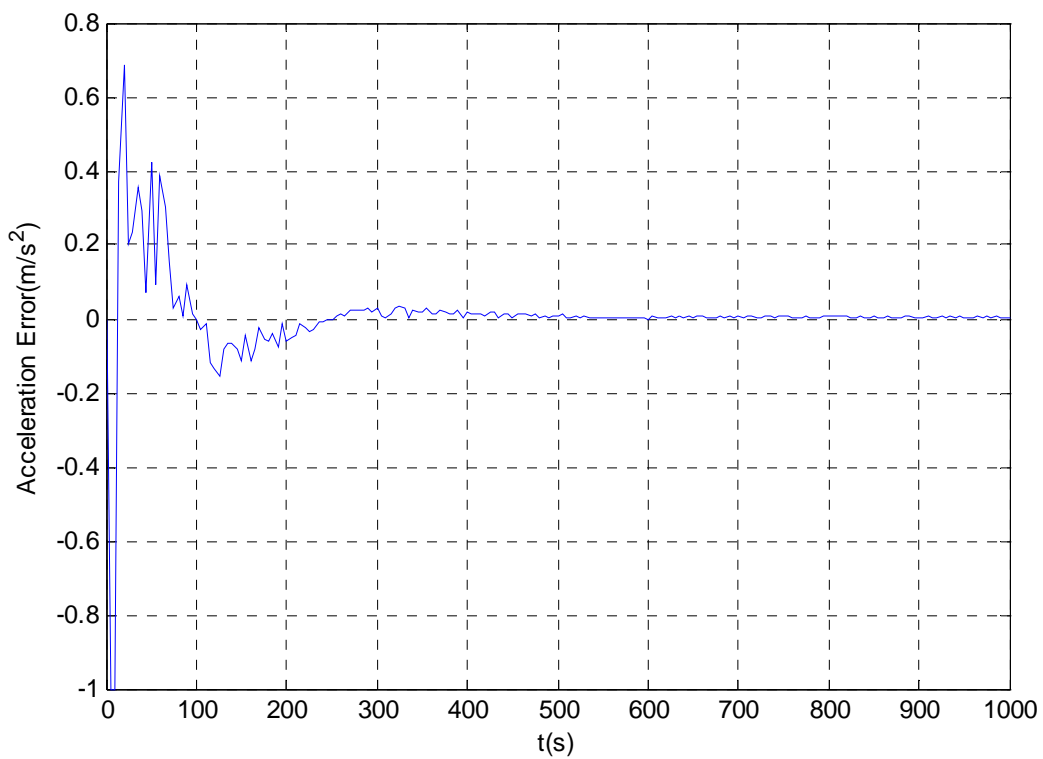

Figure 6. Fusion of the estimated GPS and accelerator data using the proposed methodology

The performance of the proposed fusion method in terms of fusion of heterogeneous sensor data has also been evaluated in comparison with the Kalman fusion method. Experiments have been conducted under the same conditions as Fig. 5 and Fig. 6 by utilizing the Kalman fusion method. Fig. 7 shows the results estimated from a single sensor (the GPS) by the Kalman 
method. The maximum error within the time period from $200 s$ to $600 s$ was found to be $0.2 \mathrm{~m} / \mathrm{s}^{2}$, and the maximum error after $600 \mathrm{~s}$ was obtained as $0.18 \mathrm{~m} / \mathrm{s}^{2}$. Comparing Fig. 5 with Fig. 7, it can be seen that the proposed methodology has much higher accuracy than the Kalman method in terms of the single sensor estimation. Fig. 8 illustrates the results for the Kalman fusion of the data estimated from both the GPS and accelerometer. The maximum error within the time period from $200 \mathrm{~s}$ to $600 \mathrm{~s}$ was found to be $0.07 \mathrm{~m} / \mathrm{s}^{2}$, and the maximum error after $600 s$ was obtained as $0.03 m / s^{2}$. Comparing Fig. 6 with Fig. 8, it can be seen that the accuracy of fusion estimation achieved by the proposed methodology is much higher than that by the Kalman method. Based on the above experimental results, it is evident that the proposed methodology has higher accuracy in fusion of heterogeneous sensor data than the Kalman fusion method.

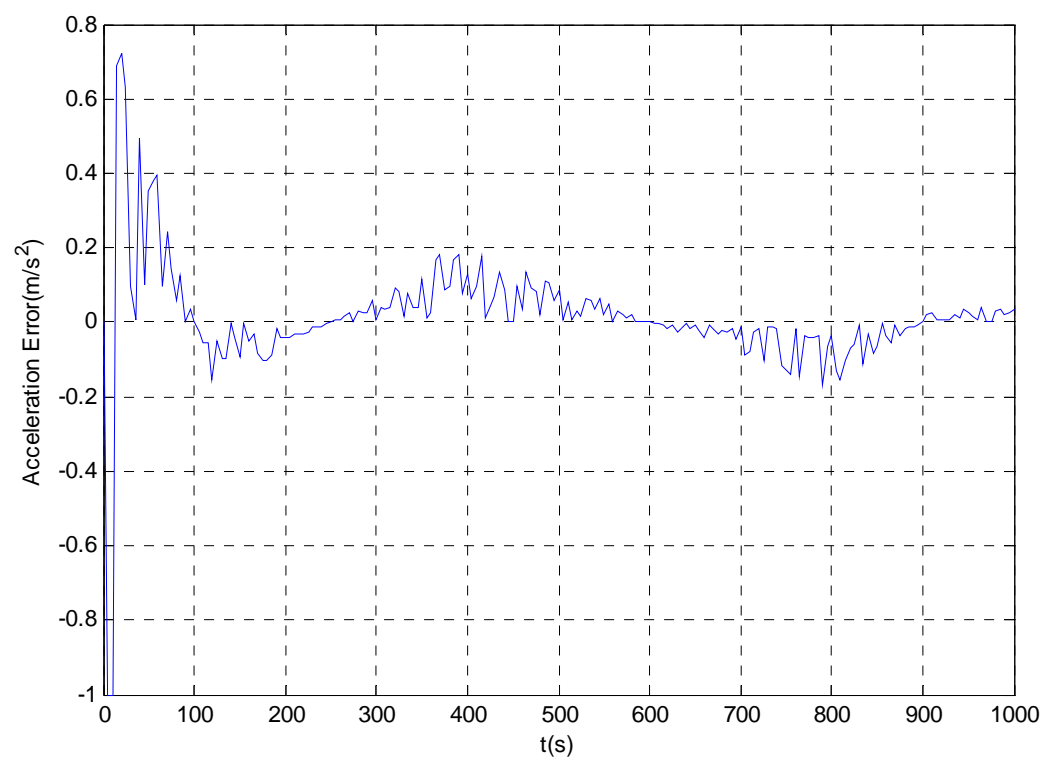

Figure 7. Kalman estimation of the GPS data 


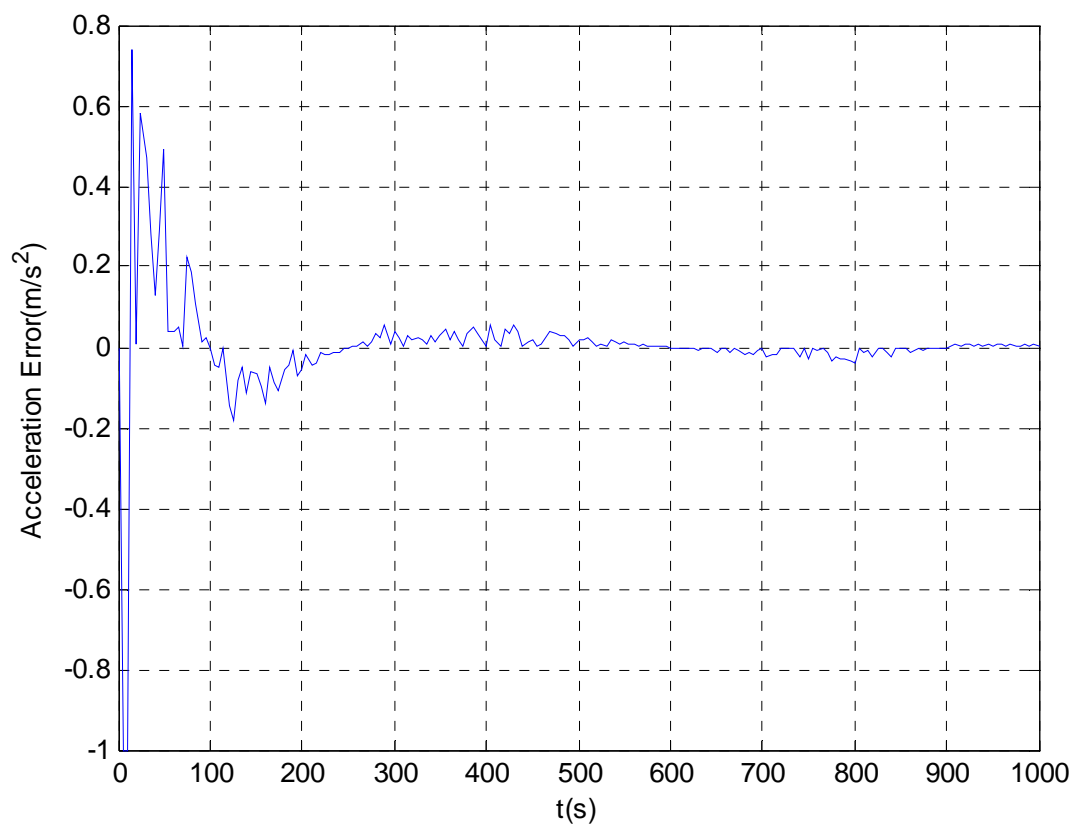

Figure 8. Kalman fusion of the data estimated from the GPS and accelerometer

From the above experiments, it can be seen that the proposed random weighting methodology can effectively fuse position data of a multi-sensor system. The accuracy is significantly improved by fusion estimation in comparison with single sensor estimation. The fusion accuracy of the proposed methodology is also much higher than that of the Kalman filter.

\section{Conclusions}

This paper presents a random weighting estimation methodology for fusion of multi-dimensional position data. The contribution of the paper is that the theory and algorithm of random weighting estimation are established for optimal fusion of multi-dimensional position data. Experiments and comparison analysis demonstrate that the proposed methodology can effectively estimate position parameters of a multi-sensor system, and the fusion accuracy of the proposed methodology is much higher than that of the Kalman filter. 
Future research work will mainly focus on intelligent multi-sensor data fusion. The proposed random weighting methodology will be combined with advanced expert systems and neural networks, and thus establishing intelligent random weighting methodologies for automatic fusion of multi-sensor data.

\section{Acknowledgements}

This research was supported by the Aeronautical Science Foundation (China) (Project Number: 20080818004) and the Natural Science Foundation of Shaanxi Province (China) (Project Number: SJ08F04).

\section{References}

1. N. A. Carlson, Federated square root filter for decentralized parallel processes, IEEE Transactions on Aerospace and Electronic Systems 26(3), 1990, pp517-525.

2. Z.-L. Deng, Y. Gao, L. Mao, Y. Li, G. Hao, New approach to information fusion steady-state Kalman filtering, Automatica 41(10), 2005, pp1695-1707.

3. D. Fox, J. Hightower, L. Liao, D. Schulz, G. Borriello, Bayesian filtering for location estimation, IEEE Pervasive Computing Magazine 2(3), 2003, pp24-33.

4. S. Gao, Z. Feng, Y. Zhong, B. Shirinzadeh, Random weighting estimation of parameters in generalized Gaussian distribution, Information Sciences 178(9), 2008, pp2275-2281.

5. S. Gao, Z. Zhang, B. Yang, The random weighting estimate of quantile process, Information Sciences 164(1-4), 2004, pp139-146.

6. S. Gao, J. Zhang, T. Zhou, Law of large number for sample mean of random weighting estimate, Information Sciences 155(1-2), 2003, pp151-156.

7. S. Gao, Y. Zhong, Random weighting estimation of kernel density, Journal of Statistical Planning and Inference 140(9), 2010, pp2403-2407.

8. S. Gao, Y. Zhong, X. Zhang, B. Shirinzadeh, Multi-sensor data fusion for INS/GPS/SAR integrated navigation system, Aerospace Science and Technology 13(4-5), 2009, pp232-237.

9. Q. Guo, S. Chen, H. Leung, S. Liu, Covariance intersection based image fusion technique with application to pansharpening in remote sensing, Information Sciences, 180(18), 2010, pp3434-3443.

10. R. Kumar, M. Wolenetz, B. Agarwalla, A framework for distributed data fusion, Information Fusion 8(3), 2007, pp227-251. 
11. J. Ma, J. Zhang, J. Yang, N. Zhang, An improved information fusion algorithm based SVM, IEEE International Conference on Computational Intelligence and Security Workshops, Harbin, China, 2007, pp397-400.

12. A. Makarenko, H. Durrant-Whyte, Decentralized Bayesian algorithms for active sensor networks, Information Fusion 7(4), 2006, pp418-433.

13. L. I. Perlovsky, Cognitive high level information fusion, Information Sciences, 177(10), 2007, pp2099-2118.

14. A. L. Ralescu, D. A. Ralescu, Y. Yamakata, Inference by aggregation of evidence with applications to fuzzy probabilities, Information Sciences, 177(2), 2007, pp378-387

15. S.-L. Sun, Multi-sensor optimal fusion fixed-interval Kalman smoothers, Information Fusion 9(2), 2008, pp293-299.

16. S.-L. Sun, Z.-L. Deng, Multi-sensor optimal information fusion Kalman filter, Automatica 40(6), 2004, pp1017-1023.

17. R. R. Yager, A framework for multi-source data fusion, Information Sciences, 163(1-3), 2004, pp175-200.

18. R. R. Yager, A framework for reasoning with soft information, Information Sciences, 180(?), 2010, pp1390-1406.

19. G. Yang, Y. Lin, P. Bhattacharya, A driver fatigue recognition model based on information fusion and dynamic Bayesian network, Information Sciences, 180(?), 2010, pp1942-1954.

20. Z. Zheng, Random weighting method, Acta Mathematicae Applicatae Sinica 10(2), 1987, pp247-253. 